\title{
Psychomotor Intervention Using Biofeedback Technology for the Elderly with Chronic Obstructive Pulmonary Disease
}

International Workshop on Gerontechnology

IWoG 2018: Gerontechnology pp 216-221 | Cite as

- Maria Santos (1)

- Rafaela Moreira (1)

- Ricardo Saldanha (1) Email author (saldanhao5@gmail.com)

- Salomé Palmeiro (1)

- César Fonseca (2) View author's OrcID profile (View OrcID profile)

1. Évora University, , Évora, Portugal

2. Évora University, Investigator POCTEP 0445_4IE_4_P, , Évora, Portugal

Conference paper

First Online: 14 April 2019

- 240 Downloads

Part of the Communications in Computer and Information Science book series (CCIS, volume 1016)

\section{Abstract}

When we talk about aging we must take into account not only chronological age, but also a complex and dynamic process of biological, psychological and social changes. Chronic Obstructive Pulmonary Disease (COPD) is highlighted in this study, as it is one of the most common and one of the leading causes of worldwide mortality in the elderly. Thus, the importance of psychomotricity in this case, since it acts on the awareness and regulation of the physiological and psychological systems, facilitating the learning process (according to the principles of learning theory and cognitive behavioral). In addition, it is also proposed the use of support technology based on the therapeutic strategies of the biofeedback system. Therefore, through the application of the goals proposed by the psychomotricity and use of the technology associated with biofeedback, the individual with COPD becomes capable of controlling the symptoms associated with the disease, and consequently acquiring more autonomy in the daily life (essential to promote their quality of life).

\section{Keywords}

Elderly Chronic Obstructive Pulmonary Disease Psychomotricity

Respiratory disturbances Biofeedback 
This is a preview of subscription content, $\underline{\log } \underline{\text { in }}$ to check access.

\section{References}

Costa, A.M.P.: Analysis of pulmonary airflow on a smartphone application (2016) Google Scholar (https://scholar.google.com/scholar?

q=Costa\%2C\%20A.M.P.\%3A\%20Analysis\%20of\%2opulmonary\%20airflow\%20on\%2 oa\%20smartphone\%20application\%20\%282016\%29)

DGS (Direcção Geral de Saúde): Programa Nacional de Prevenção e Controlo da Doença Pulmonar Obstrutiva Crónica. Lisboa: DGS (2005).

https://www.dgs.pt/documentos-epublicacoes/programa-nacional-de-prevencao-econtrolo-da-doenca-pulmonar-obstrutiva-cronica-pdf.aspx

(https://www.dgs.pt/documentos-epublicacoes/programa-nacional-de-prevencao-econtrolo-da-doenca-pulmonar-obstrutiva-cronica-pdf.aspx). acedido em 2018.

Journal 2(5), 99-110 (2016)

Fonseca, V., Martins, R.F.R.: Progressos em psicomotricidade (2001)

Google Scholar (https://scholar.google.com/scholar?

q=Fonseca\%2C\%2oV.\%2C\%20Martins\%2C\%2OR.F.R.\%3A\%2oProgressos\%2oem\%20 psicomotricidade\%20\%282001\%29)

Kawagoshi, A., et al.: Effects of low-intensity exercise and home-based pulmonary rehabilitation with pedometer feedback on physical activity in elderly patients with chronic obstructive pulmonary disease. Respir. Med. 109(3), 364-371 (2015)

CrossRef (https://doi.org/10.1016/j.rmed.2015.01.008)

Google Scholar (http://scholar.google.com/scholar_lookup?

title=Effects\%20of\%2olow-intensity\%20exercise\%20and\%2ohome-

based\%2opulmonary\%2orehabilitation\%20with\%2opedometer\%2ofeedback\%20on\% 2ophysical\%20activity\%20in\%20elderly\%2opatients\%20with\%2ochronic\%20obstruc tive\%2opulmonary\%20disease\&author=A.\%20Kawagoshi\&journal=Respir.\%20Med. \&volume $=109 \&$ issue $=3 \&$ pages $=364-371 \&$ publication_year $=2015$ )

Matos, M.L.P.D.: Efeito de dois programas de reabilitação psicomotora (atividade contínua versus atividade intervalada) na capacidade neuromotora de idosos institucionalizados. Master's thesis, Universidade de Évora (2016)

Google Scholar (https://scholar.google.com/scholar?

q=Matos\%2C\%20M.L.P.D.\%3A\%20Efeito\%20de\%2odois\%2oprogramas\%2ode\%2ore abilita\%C3\%A7\%C3\%A30\%2opsicomotora\%20\%28atividade\%2ocont\%C3\%ADnua\%2 oversus\%20atividade\%2ointervalada\%29\%20na\%20capacidade\%2oneuromotora\%20 de\%20idosos\%2oinstitucionalizados.\%2oMaster\%E2\%80\%99s\%20thesis\%2C\%2oUni versidade\%20de\%20\%C3\%89vora\%20\%282016\%29)

McCarthy, B., Casey, D., Devane, D., Murphy, K., Murphy, E., Lacasse, Y.: Pulmonary rehabilitation for chronic obstructive pulmonary disease. The Cochrane Library (2015) Google Scholar (https://scholar.google.com/scholar?

q=McCarthy\%2C\%2oB.\%2C\%20Casey\%2C\%20D.\%2C\%2oDevane\%2C\%20D.\%2C\%2 oMurphy\%2C\%2oK.\%2C\%2oMurphy\%2C\%2oE.\%2C\%2oLacasse\%2C\%2OY.\%3A\%2O Pulmonary\%2orehabilitation\%2ofor\%20chronic\%20obstructive\%2opulmonary\%2odi sease.\%20The\%2oCochrane\%2oLibrary\%20\%282015\%29)

Neto, A.R.N.: Biofeedback em terapia cognitivo-comportamental. Arq. Méd. Hosp.

Fac. Ciênc. Méd. Santa Casa São Paulo 55(3), 127-132 (2018)

Google Scholar (http://scholar.google.com/scholar_lookup?

title=Biofeedback\%20em\%20terapia\%20cognitivo-

comportamental\&author=ARN.\%20Neto\&journal=Arq.\%20M\%C3\%A9d.\%20Hosp.\% 
20Fac.\%20Ci\%C3\%AAnc.\%20M\%C3\%A9d.\%2oSanta\%2oCasa\%2oS\%C3\%A30\%20Pa ulo\&volume $=55 \&$ issue $=3 \&$ pages $=127-132 \&$ publication_year $=2018)$

Phipps, W.J.: Enfermagem Médico-Cirúrgica, Conceitos e Prática Clínica, 6a Edição. Lusodidacta (Livro) (2004)

Google Scholar (https://scholar.google.com/scholar?

q=Phipps\%2C\%2oW.J.\%3A\%2oEnfermagem\%2oM\%C3\%A9dico-

Cir\%C3\%BArgica\%2C\%2oConceitos\%20e\%2oPr\%C3\%A1tica\%2oCl\%C3\%ADnica\%2C \%206\%C2\%AA\%20Edi\%C3\%A7\%C3\%A30.\%2OLusodidacta\%20\%28Livro\%29\%20\%2 $82004 \% 29)$

Rissardi, G.G., Godoy, M.F.: Estudo da aplicação da técnica de relaxamento muscular progressivo de Jacobson modificada nas respostas das variáveis cardiovasculares e respiratórias de clientes hansenianos. Arq. Ciênc. Saúde 14(3), 175-180 (2004)

Google Scholar (http://scholar.google.com/scholar_lookup?

title=Estudo\%20da\%20aplica\%C3\%A7\%C3\%A30\%20da\%20t\%C3\%A9cnica\%20de\%2 orelaxamento\%20muscular\%2oprogressivo\%20de\%20Jacobson\%20modificada\%20n as\%2orespostas\%20das\%2Ovari\%C3\%A1veis\%2ocardiovasculares\%20e\%2orespirat\% C3\%B3rias\%2ode\%2oclientes\%2ohansenianos\&author=GG.\%20Rissardi\&author=M F.\%20Godoy\&journal=Arq.\%20Ci\%C3\%AAnc.\%20Sa\%C3\%BAde\&volume=14\&issue= 3\&pages $=175-180 \&$ publication_year $=2004$ )

Ruivo, S., Viana, P., Martins, C., Baeta, C.: Efeito do envelhecimento cronológico na função pulmonar. Comparação da função respiratória entre adultos e idosos saudáveis. Rev. Port. Pneumol. 15(4), 629-653 (2009)

CrossRef (https://doi.org/10.1016/So873-2159(15)30161-6)

Google Scholar (http://scholar.google.com/scholar_lookup?

title=Efeito\%20do\%2oenvelhecimento\%20cronol\%C3\%B3gico\%2ona\%2ofun\%C3\%A 7\%C3\%A30\%2opulmonar.\%20Compara\%C3\%A7\%C3\%A30\%20da\%2ofun\%C3\%A7\% $\mathrm{C}_{3} \% \mathrm{~A} 30 \%$ 2orespirat\%C3\%B3ria\%2oentre\%20adultos\%20e\%2oidosos\%2osaud\%C3\% A1veis\&author $=$ S.\%20Ruivo\&author $=$ P.\%20Viana\&author $=$ C.\%20Martins\&author $=$ C. \%20Baeta\&journal=Rev.\%20Port.\%20Pneumol.\&volume=15\&issue $=4$ \&pages=629-

653\&publication_year=2009)

Schwartz, M.S., Andrasik, F.: Biofeedback: A practitioner's guide. Guilford

Publications (2017)

Google Scholar (https://scholar.google.com/scholar?

q=Schwartz\%2C\%2OM.S.\%2C\%2OAndrasik\%2C\%20F.\%3A\%2oBiofeedback\%3A\%20 A\%2opractitioner\%E2\%80\%99s\%20guide.\%20Guilford\%20Publications\%20\%28201 7\%29)

Spruit, M.A., Pitta, F., McAuley, E., ZuWallack, R.L., Nici, L.: Pulmonary rehabilitation and physical activity in patients with chronic obstructive pulmonary disease. Am. J.

Respir. Crit. Care Med. 192(8), 924-933 (2015)

CrossRef (https://doi.org/10.1164/rccm.201505-0929CI)

Google Scholar (http://scholar.google.com/scholar_lookup?

title=Pulmonary\%2orehabilitation\%20and\%2ophysical\%20activity\%20in\%2opatients \%20with\%20chronic\%20obstructive\%2opulmonary\%2odisease\&author=MA.\%20Spr uit\&author=F.\%20Pitta\&author=E.\%20McAuley\&author=RL.\%20ZuWallack\&author =L.\%2oNici\&journal=Am.\%20J.\%2oRespir.\%20Crit.\%20Care\%2oMed.\&volume=192 \&issue $=8 \&$ pages $=924-933 \&$ publication_year $=2015$ )

Bongaarts, J.: United Nations, Department of Economic and Social Affairs, Population Division, Sex Differentials in Childhood Mortality. Popul. Dev. Rev. 4o(2), 380 (2014) CrossRef (https://doi.org/10.1111/j.1728-4457.2014.00686.x)

Google Scholar (http://scholar.google.com/scholar_lookup?

title=United\%20Nations\%2C\%20Department\%20of\%20Economic\%20and\%20Social 
\%20Affairs\%2C\%20Population\%2oDivision\%2C\%20Sex\%2oDifferentials\%2oin\%20C hildhood\%20Mortality\&author=J.\%20Bongaarts\&journal=Popul.\%2oDev.\%20Rev.\&v olume $=40 \&$ issue $=2 \&$ pages $=380 \&$ publication $\_$year $=2014 ）$

Burke, D., Gorman, E., Stokes, D., Lennon, O.: An evaluation of neuromuscular electrical stimulation in critical care using the ICF framework: a systematic review and meta-analysis. Clin. Respir. J. (2014)

Google Scholar (https://scholar.google.com/scholar?

q=Burke\%2C\%20D.\%2C\%20Gorman\%2C\%20E.\%2C\%2OStokes\%2C\%2oD.\%2C\%2OL ennon\%2C\%20O.\%3A\%20An\%2oevaluation\%20of\%2oneuromuscular\%2oelectrical\% 20stimulation\%20in\%20critical\%20care\%2ousing\%2othe\%20ICF\%2oframework\%3A \%20a\%20systematic\%2oreview\%20and\%20metaanalysis.\%20Clin.\%20Respir.\%20J.\%20\%282014\%29)

Direcção-Geral da Saúde (DGS) Translation of: World Health Organization (resolution WHA54.21). International classification of functioning, disability and health (2004) Google Scholar (https://scholar.google.com/scholar?q=Direc\%C3\%A7\%C3\%A30Geral\%20da\%20Sa\%C3\%BAde\%20\%28DGS\%29\%20Translation\%20of\%3A\%20Worl d\%20Health\%20Organization\%20\%28resolution\%2OWHA54.21\%29.\%2OInternation al\%2oclassification\%20of\%2ofunctioning\%2C\%20disability\%20and\%2ohealth\%20\% 282004\%29)

European Commission (EC): Population, Key figures on Europe - 2014 edn. Publications Office of the European Union, Luxembourg (2014). ISSN 2315-201X Google Scholar (https://scholar.google.com/scholar? q=European\%20Commission\%20\%28EC\%29\%3A\%20Population\%2C\%2oKey\%2ofig ures\%20on\%2oEurope\%20\%E2\%80\%93\%202014\%20edn.\%20Publications\%2oOffic e\%20of\%20the\%20European\%20Union\%2C\%20Luxembourg\%20\%282014\%29.\%20I SSN\%202315-201X)

Florin, J., Ehrenberg, A., Ehnfors, M., Björvell, C.: A comparison between the VIPS model and the ICF for expressing nursing content in the health care record. Int. J.

Med. Inform. 82(2), 108-117 (2013)

CrossRef (https://doi.org/10.1016/j.ijmedinf.2012.05.016)

Google Scholar (http://scholar.google.com/scholar_lookup?

title=A\%20comparison\%2obetween\%20the\%20VIPS\%20model\%20and\%20the\%20I CF\%2ofor\%20expressing\%2onursing\%2ocontent\%2oin\%20the\%2ohealth\%20care\%2 orecord\&author=J.\%20Florin\&author=A.\%20Ehrenberg\&author=M.\%20Ehnfors\&au thor $=\mathrm{C} . \% 20 \mathrm{Bj} \% \mathrm{C} 3 \%$ B6rvell\&journal=Int.\%20J.\%20Med.\%20Inform.\&volume=82\&is sue $=2 \&$ pages $=108-117 \&$ publication_year $=2013$ )

José Lopes, M., et al.: Evaluation of elderly persons' functionality and care needs. Rev. Lat. Am. Enferm. (RLAE) 21, 52-6o (2013)

CrossRef (https://doi.org/10.1590/So104-11692013000700008)

Google Scholar (http://scholar.google.com/scholar_lookup?

title=Evaluation\%20of\%2oelderly\%2opersons\%E2\%80\%99\%2ofunctionality\%20and \%20care\%20needs\&author=M.\%20Jos\%C3\%A9\%20Lopes\&journal=Rev.\%2OLat.\%20 Am.\%20Enferm.\%20\%28RLAE\%29\&volume $=21$ \&pages $=52-$

60\&publication_year=2013)

Maric, M., de Haan, E., Hogendoorn, S.M., Wolters, L.H., Huizenga, H.M.: Evaluating statistical and clinical significance of intervention effects in single-case experimental designs: an SPSS method to analyse univariate data. Behav. Therapy 46(2), 230-241 (2015)

CrossRef (https://doi.org/10.1016/j.beth.2014.09.005)

Google Scholar (http://scholar.google.com/scholar_lookup?

title=Evaluating\%2ostatistical\%20and\%20clinical\%2osignificance\%20of\%2ointervent 
ion\%20effects\%20in\%20single-

case\%2oexperimental\%2odesigns\%3A\%20an\%20SPSS\%2omethod\%2oto\%20analyse \%20univariate\%2Odata\&author=M.\%2OMaric\&author=E.\%2OHaan\&author=SM.\%2O Hogendoorn\&author=LH.\%2oWolters\&author=HM.\%2oHuizenga\&journal=Behav.\% 20Therapy\&volume $=46 \&$ issue $=2 \&$ pages $=230-241 \&$ publication_year $=2015$ )

Marôco, J.: Análise Estatística com Utilisação do SPSS [Statistical Analysis using SPSS], 6th edn. Edições Silabo, p. 990 (2014). ISBN: 9789899676343

Google Scholar (https://scholar.google.com/scholar?

$\mathrm{q}=\mathrm{Mar} \% \mathrm{C}_{3} \% \mathrm{~B} 4 \mathrm{co} \% 2 \mathrm{C} \% 20 J . \% 3 \mathrm{~A} \% 20 \mathrm{An} \% \mathrm{C}_{3} \%$ A1lise\%2oEstat\%C3\%ADstica\%2ocom \%20Utilisa\%C3\%A7\%C3\%A30\%20do\%20SPSS\%20\%5BStatistical\%2oAnalysis\%2ousi ng\%20SPSS\%5D\%2C\%206th\%2Oedn.\%2OEdi\%C3\%A7\%C3\%B5es\%2oSilabo\%2C\%20 p.\%20990\%20\%282014\%29.\%20ISBN\%3A\%209789899676343)

Meskell, P., Murphy, K., Shaw, D.G., Casey, D.: Insights into the use and complexities of the Policy Delphi technique. Nurse Res. 21(3), 32-39 (2014)

CrossRef (https://doi.org/10.7748/nr2014.01.21.3.32.e342)

Google Scholar (http://scholar.google.com/scholar_lookup?

title=Insights\%20into\%20the\%20use\%20and\%20complexities\%20of\%20the\%20Polic y\%20Delphi\%2otechnique\&author=P.\%20Meskell\&author=K.\%20Murphy\&author= DG.\%20Shaw\&author=D.\%20Casey\&journal=Nurse\%20Res.\&volume=21\&issue $=3 \& p$ ages $=32-39 \&$ publication_year $=2014$ )

Orem, D.E.: Nursing: Concepts of Practice, 6th edn. Mosby, St. Louis (2001)

Google Scholar (http://scholar.google.com/scholar_lookup?

title=Nursing\%3A\%20Concepts\%20of\%20Practice\&author=DE.\%20Orem\&publicatio n_year=2001)

Pereira, C., Fonseca, C., Escoval, A., Lopes, M.: Contributo para a classificação da funcionalidade na população com mais de 65 anos, segundo a classificação internacional de funcionalidade. Rev. Port. Saúde Pública 19(1), 52-62 (2011) Google Scholar (http://scholar.google.com/scholar_lookup?

title=Contributo\%2opara\%20a\%20classifica\%C3\%A7\%C3\%A30\%20da\%2ofuncionali dade\%2ona\%2opopula\%C3\%A7\%C3\%A30\%20com\%20mais\%20de\%2065\%20anos\% 2C\%20segundo\%20a\%2oclassifica\%C3\%A7\%C3\%A30\%2ointernacional\%2ode\%2ofu ncionalidade\&author $=\mathrm{C} . \% 20$ Pereira\&author $=\mathrm{C} . \% 20$ Fonseca\&author $=\mathrm{A} . \% 20$ Escoval $\&$ author=M.\%2oLopes\&journal=Rev.\%20Port.\%20Sa\%C3\%BAde\%2oP\%C3\%BAblica\& volume $=19$ \&issue $=1$ \&pages $=52-62$ \&publication $\_$year $=2011$ )

Shen, P., Chen, C.: The WHO's international classification of functioning, disability, and health (ICF): essential knowledge for nurses. Hu Li Za Zhi J. Nurs. 59(6), 92-97 (2012)

Google Scholar (http://scholar.google.com/scholar_lookup?

title=The\%20WHO\%E2\%80\%99s\%20international\%20classification\%20of\%2ofuncti oning\%2C\%2odisability\%2C\%20and\%2ohealth\%20\%28ICF\%29\%3A\%20essential\%2 oknowledge\%2ofor\%2onurses\&author=P.\%20Shen\&author=C.\%20Chen\&journal=H u\%20Li\%20Za\%20Zhi\%20J.\%20Nurs.\&volume=59\&issue=6\&pages=92-

97\&publication_year=2012)

Sidani, S.: Effects of patient-centered care on patient outcomes: an evaluation. Res.

Theory Nurs. Pract. 22(1), 24-37 (2008)

CrossRef (https://doi.org/10.1891/1541-6577.22.1.24)

Google Scholar (http://scholar.google.com/scholar_lookup?

title=Effects\%20of\%2opatient-

centered\%20care\%20on\%2opatient\%20outcomes\%3A\%20an\%20evaluation\&author= S.\%20Sidani\&journal=Res.\%20Theory\%20Nurs.\%20Pract.\&volume=22\&issue=1\&pag es $=24-37 \&$ publication_year $=2008$ ) 
So, H., Kim, H., Ju, K.: Prediction model of quality of life in elderly based on ICF model. J. Korean Acad. Nurs. 41(4), 481-490 (2011)

CrossRef (https://doi.org/10.4040/jkan.2011.41.4.481)

Google Scholar (http://scholar.google.com/scholar_lookup?

title=Prediction\%20model\%20of\%20quality\%20of\%2olife\%2oin\%20elderly\%2obase d\%200n\%20ICF\%20model\&author=H.\%2OSo\&author=H.\%2OKim\&author=K.\%2OJu \&journal=J.\%20Korean\%20Acad.\%20Nurs.\&volume $=41 \&$ issue $=4 \&$ pages $=481$ 490\&publication_year=2011)

The United Nations 2012 Population Projections. (UN) Population \& Development Review 39(3), 551-555 (2013)

Google Scholar (https://scholar.google.com/scholar? q=The\%20United\%20Nations\%202012\%20Population\%20Projections.\%20\%28UN\% 29\%20Population\%20\%26\%2oDevelopment\%20Review\%2039\%283\%29\%2C\%20551 \%Е2\%80\%93555\%20\%282013\%29)

World Bank (WB): The World Bank Annual Report 2014: Year in Review, p. 67 (2014) Google Scholar (https://scholar.google.com/scholar? q=World\%20Bank\%20\%28WB\%29\%3A\%20The\%20World\%20Bank\%20Annual\%20 Report\%202014\%3A\%20Year\%20in\%20Review\%2C\%20p.\%2067\%20\%282014\%29)

Zhu, H.: Correctly selection methods of building up a model function of known categories in SPSS 16.0. J. Cap. Inst. Phys. Educ. 26(1), 91-96 (2014)

Google Scholar (http://scholar.google.com/scholar_lookup?

title=Correctly\%20selection\%2omethods\%20of\%2obuilding\%20up\%20a\%20model\% 2ofunction\%20of\%2oknown\%20categories\%20in\%20SPSS\%2016.o\&author=H.\%2OZ hu\&journal=J.\%20Cap.\%20Inst.\%20Phys.\%20Educ.\&volume=26\&issue=1\&pages=91 -96\&publication_year=2014)

\section{Copyright information}

(C) Springer Nature Switzerland AG 2019

\section{About this paper}

Cite this paper as:

Santos M., Moreira R., Saldanha R., Palmeiro S., Fonseca C. (2019) Psychomotor Intervention Using Biofeedback Technology for the Elderly with Chronic Obstructive Pulmonary Disease. In: García-Alonso J., Fonseca C. (eds) Gerontechnology. IWoG 2018. Communications in Computer and Information Science, vol 1016. Springer, Cham

- First Online 14 April 2019

- DOI https://doi.org/10.1007/978-3-030-16028-9_19

- Publisher Name Springer, Cham

- Print ISBN 978-3-030-16027-2

- Online ISBN 978-3-030-16028-9

- eBook Packages Computer Science

- Buy this book on publisher's site

- Reprints and Permissions

\section{Personalised recommendations}




\section{SPRINGER NATURE}

(C) 2019 Springer Nature Switzerland AG. Part of $\underline{\text { Springer Nature. }}$

Not logged in Not affiliated 85.243.120.45 